\title{
Acquired pendular nystagmus: its characteristics, localising value and pathophysiology
}

\author{
MA GRESTY, JJ ELL, LJ FINDLEY \\ From the Medical Research Council, Neuro-Otology Unit, Institute of Neurology, The National Hospital, \\ London, $U K$
}

SUMMARY Investigations were made of 16 patients with acquired pendular nystagmus and a further 32 cases reported in the literature were reviewed. Amongst our own patients two thirds had multiple sclerosis, almost one third a cerebrovascular accident or angioma and two had optic atrophy with squint. The nystagmus took forms which could be monocular or binocular, conjugate or disconjugate and could involve movements about single or multiple axes. Spectral analysis was used to characterise the amplitude and frequency of the movements and to estimate the degree of relationship (coherence) between movements of the two eyes or between movements of one eye about several axes. The oscillations ranged in frequency from $2.5 \mathrm{~Hz}$ to $6 \mathrm{~Hz}$, with typical amplitudes between $3^{\circ}$ and $5^{\circ}$. In a given patient all oscillations, regardless of plane, were highly synchronised. Somatic tremors of the upper limb, face and palate associated with the nystagmus were often at similar frequencies to the eye movement. The other ocular signs common to all our patients were the presence of squint with failure of convergence. Most patients also had skew deviation or internuclear ophthalmoplegia or both. The major oculomotor systems, that is, saccades, pursuit, optokinetic and vestibulo-ocular reflexes could be intact. It is inferred that the mechanism responsible for the pendular nystagmus lies at a level which is close to the oculomotor nuclei so that it can have monocular effects but is not part of the primary motor pathways. It is possible that this mechanism normally subserves maintenance of conjugate movement and posture of the eyes. The periodicity of the nystagmus is likely to arise from instability in a certain type(s) of neurone, for the associated somatic tremors have similar characteristics and yet involve very different neuronal muscular circuitry. Prognosis for cessation of the nystagmus is poor. In five patients with multiple sclerosis it was suppressed by intravenous hyoscine with, however, unacceptable subsequent side effects.

"These eyes that rowle in vain

To find thy piercing ray, and find no dawn." John Milton, Paradise Lost, III, 11/23-24

Pendular nystagmus is a sinusoidal, involuntary oscillation of the eyes which may be congenital or acquired in nervous diseases. Clinically, differentiation between congenital and acquired nystagmus is not difficult because of their distinct characteristics. Congenital pendular nystagmus is horizontal, binocular and conjugate, can have a variable wave

Address for reprint requests: Dr MA Gresty, the Institute of Neurology, Queen Sq, London WC1N 3BG, UK

Received 6 November 1981. Accepted 17 January 1982 form and changes into a jerk nystagmus on lateral gaze. Acquired pendular nystagmus may be monocular or binocular, conjugate or disconjugate with a wave form which is largely independent of eye position. The movement of the eye may be vertical, horizontal or torsional in which case the globe rotates about the principal axis. An observed movement may be the result of combined oscillations about more than one axis as in "rotary" nystagmus (fig 1). Pendular nystagmus sometimes occurs in association with rhythmical movements at similar frequencies of other parts of the body, as in the syndrome of "oculo-palatal-myoclonus" which postmortem examination has confirmed a lesion in the brain stem. The nystagmus in this syn431 


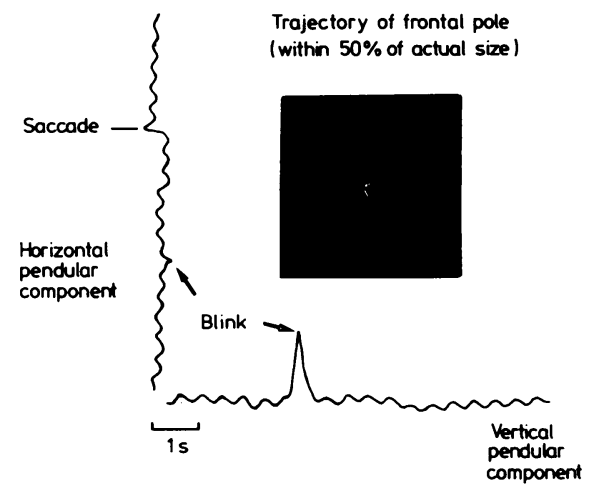

Fig 1 An example of monocular rotatory nystagmus recorded from a 25-year-old male patient with multiple sclerosis. The rotation was the result of synchronous pendular oscillations in both the vertical and horizontal planes. The central figure indicating the trajectory was produced by applying the horizontal and vertical electro-oculographic recordings to the $y$ and $x$ amplifiers of an oscilloscope. The maintenance of the unchanging elliptical trajectory indicates the precision with which horizontal and vertical components were synchronised.

drome is always similar to the form which can occur in isolation and there is no reason to suspect that they have separate origins.

Although the planes in which the eyes move and the phase relationships between the movements show considerable variability from one case to another there are features common to acquired pendular nystagmus which lead us to suspect that the movements should be classified together. Firstly, both within and between patients the frequency and amplitudes of the nystagmus are similar giving a clinical appearance which is quite distinctive regardless of the plane of movement. In addition, in any individual, nystagmus which occurs in disconjugate planes is synchronised indicating some form of yoking between vertical, horizontal and torsional oculomotor systems. These features suggest that a unitary mechanism or at least similar types of mechanisms sharing a common rhythm generator are responsible for the various manifestations of pendular nystagmus.

In the only extensive survey of acquired pendular nystagmus to date $e^{2}$ the authors postulated that the ocular movements originated from the cerebellar nuclei and their connections and were evidence of a failure to maintain eye posture. The existence of monocular pendular nystagmus throws doubt upon this theory. ${ }^{3}$ Accordingly we examined 16 patients with acquired pendular nystagmus with a particular view to determining the abnormalities of ocular movements common to all which might provide clues as to aetiology and pathophysiology.

\section{Methods}

Recordings of eye movements were taken using direct coupled electro-oculography with a low pass cut off usually set at $32 \mathrm{~Hz}$. In cases of torsional pendular nystagmus two small silver-silver chloride electrodes were placed on the upper lid over the insertion of the superior oblique muscle into the globe. The signals were amplified with a wide bandpass in order to detect the EMG activity of the oblique muscle. Accelerometric recordings of head or limb movements were taken using linear piezo resistive accelerometers (Endevco 7265-10) mounted with their sensitive axes oriented in the major plane of movement. The device had a frequency response from steady state acceleration up to a superimposed low pass cut off at $32 \mathrm{~Hz}$. Recordings of limb displacements were obtained using a Schottky barrier photo-detector mounted with its sensitive surface in the focal plane of a $35 \mathrm{~mm}$ camera which was oriented to view the limb from a suitable angle. A small pilot lamp was mounted on the limb, the movement of which was registered by the photo-detector and resolved into displacements in the vertical and horizontal planes (after the method of Findley $\mathrm{et} \mathrm{al}^{4}$ ).

The signals relating eye and body movements were subjected to spectral analysis on a Hewlett Packard 5420A digital signal analyser. The analyses employed to characterise the signals were the Auto-spectrum; used to measure the amplitude and frequency of the dominant frequency of a tremor, and Coherence (the average of square of the cross spectra divided by product of the averages of the auto-power spectra); which is a measure of the degree to which two signals (that is, tremors) are related. Coherence is expressed on a scale of 0 to 1 in which 0 indicates that the signals are independent of each other and 1 indicates that they are completely related. As a point of interpretation a coherence of 1 can be taken to indicate that the signals are causally related. These functions derived from the raw signals were averaged to improve the significance of the measurements. The analyses were performed on 1025 point digitised $10.24 \mathrm{~s}$ samples of raw data producing a maximum temporal resolution of $10 \mathrm{~ms}$, a frequency resolution of $0.05 \mathrm{~Hz}$ and bandwidth of DC to $25 \mathrm{~Hz}$. The data was digitally filtered to prevent aliasing of frequencies in excess of $25 \mathrm{~Hz}$ and windowed with a Hanning window appropriate for the analysis of sinusoidal signals. ${ }^{5}$ Averages were made on spectra derived from overlapping samples of raw data. This compensates for the reduced contribution of the data at the beginning and end of the data samples which results from the application of a Hanning window.

The validity of the raw data signals was established by displaying the signals on a storage oscilloscope and making a simultaneous comparison with an infra-red video recording of the actual eye movement. Combined vertical and horizontal movements were plotted in the form of a Lissajous figure using the $\mathrm{x}$ and $\mathrm{y}$ amplifiers of the oscilloscope (fig 1).

\section{Patients}

Sixteen patients were referred for assessment by the consultants at The National Hospital, Queen Square and 
St Thomas' Hospital, London. In addition we include data derived from a further 27 patients with multiple sclerosis reported by Aschoff et al, ${ }^{2}$ four patients described by Chokroverty and Barron ${ }^{6}$ and one of Nathanson ${ }^{7}$ and four taken from the case history records of the National Hospital who had had electro-oculographic recordings taken of their eye movements; a total of 52 patients with monocular and binocular pendular nystagmus. Congenital nystagmus of the pendular variety was excluded from the survey.

Amongst our patients 12 had clinically definite multiple sclerosis; four had had brain stem vascular accidents; one a brain stem angioma; there was one patient with congenital optic atrophy with strabismus and one with a unilateral amblyopia secondary to strabismus (both of these patients had a uniocular vertical pendular nystagmus in the affected eye); and one patient had a provisional diagnosis of brain stem encephalitis.

A universal complaint amongst these patients was one of oscillopsia which was usually sufficiently marked to impair reading ability. The majority of our patients had severe neurological disability but in the few who were ambulant and active the oscillopsia caused difficulty with daily activities. The oculomotor impairment common to all patients was some form of failure of conjugate gaze or defect of voluntary convergence.

\section{Results}

The relevant findings in our patients which relate to the behavioural characteristics of their pendular nystagmus, associated oculomotor and visual disorders, tremor of parts of the body and diagnosis are presented in the table.

\section{FREQUENCY OF PENDULAR NYSTAGMUS}

The histogram of frequencies of pendular nystagmus as measured in our own patients and as reported by other authors is presented in fig 2 . In the cases of nystagmus which varied in frequency the middle of the range of variability was used to plot the histogram. Because of the finding that in any one patient, regardless of the plane of movement, pendular nystagmus in more than one direction was phase locked, such compound nystagmus was characterised by only one frequency measurement. The frequency distribution was unimodal, skewed towards lower frequency values, with a mode at between 2.6 and $3 \mathrm{~Hz}$ with a mean at $3.6 \mathrm{~Hz}$. There was no obvious relationship between frequency and direction of nystagmus and an underlying disease process.

In any given patient, whenever an episode of well defined binocular pendular nystagmus occurred, there was a precise phase lock between the movements of the right and left eye. The phase relationship was independent of the plane of movement, which could be different in the two eyes. This characteristic is illustrated in the case of a 28-yearold female with a presumed cerebrovascular acci- dent which had left her with a binocular convergent pendular nystagmus which had remained unchanged in character for two years. An analysis of her eye movements is presented in fig 3. The raw data records in the lower part of the figure show a fluctuating pendular nystagmus of slightly greater amplitude in the left eye. The synchronisation arrow between the traces indicates that the nystagmus consisted of pendular movements of convergence and divergence. The auto-spectra of the movements of the left and right eye are averages of ten overlapping $10.24 \mathrm{~s}$ samples of eye movement recordings and indicates a frequency of $3.4 \mathrm{~Hz}$ in both eyes. The corresponding coherence function measured on the same data sample as the auto-spectra indicates a coherence of unity in the frequency band of the pendular eye movement (indicated by the single vertical arrow). The phase relationship between the right and left eye measured over the same data sample was $150^{\circ}$ indicating that the movements were not perfectly convergent but slightly staggered (a phase of $180^{\circ}$ would indicate perfect convergence). The most important of these measurements is the coherence function which increases its specificity and significance with successive averaging, unlike the other measurements in which averaging can hide the variability of the phenomena. The coherence of unity in the frequency band of the pendular movements proves that they are frequency locked.

Similar measurements on other patients with compound nystagmus revealed a similar degree of relationship between movements in the various planes. In the cases of combined horizontal and vertical oscillations the eye executes a rotation (as in fig 1) in which the circular or eilliptical pattern of the rotation is invariable indicating that frequency and phase relationships between the horizontal and vertical components of the movement are also invariable.

\section{CONCURRENT TREMOR OF OTHER PARTS OF THE} BODY

Eight patients with pendular nystagmus on whom we made measurements had tremor of other parts of the body including the head, neck and upper limbs. Of such tremors, some were similar in frequency to the pendular nystagmus and others dissimilar. Both similar and dissimilar tremor frequencies could coexist in the same person and in the same limb (depending upon whether the tremor was brought out by a posture of flexion or extension). The histogram of somatic tremor frequencies in our patients is presented in fig 3 . It is evident that the distribution of frequencies is similar to that of the pendular nystagmus.

The degree of synchronisation which can coexist 
Table Classification of diseases, ocular signs and associated somatic tremor in patients with pendular nystagmus

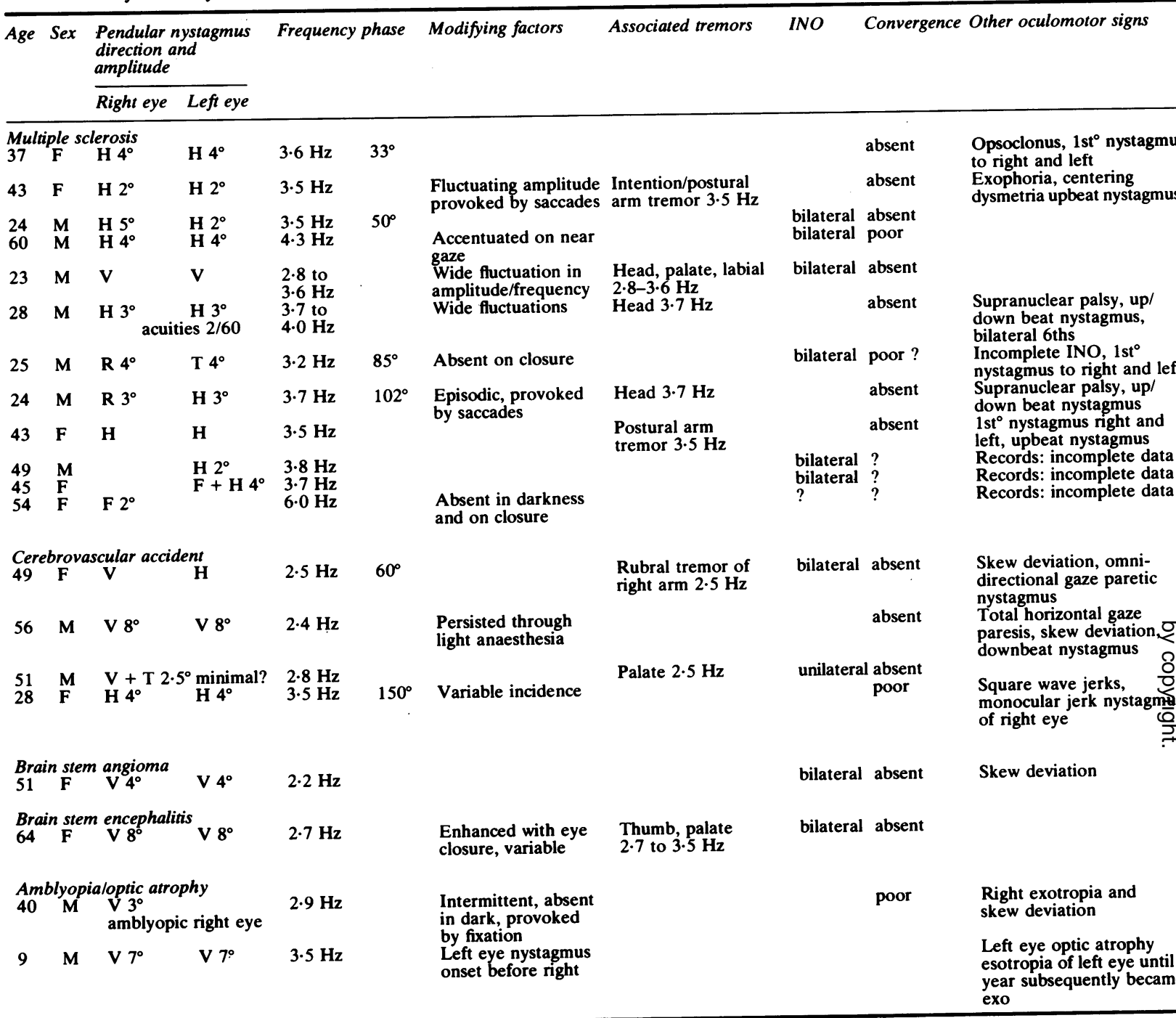

\section{$\mathbf{H}=$ horizontal, $\mathbf{V}=$ vertical, $\mathrm{T}=$ torsional}

between pendular nystagmus and simultaneous tremor of other parts of the body is illustrated in fig 4 which presents raw data records from a 23 -year-old male patient with multiple sclerosis. His nystagmus ranged in frequency from 2.8 to $3.6 \mathrm{~Hz}$ and was accompanied at various times by rhythmical movements of the head, upper lip or palate. When any combination of these occurred simultaneously they were approximately synchronous as indicated by the vertical synchronisation arrows in fig 4. Careful measurement of the traces, however, revealed that over several cycles the various tremors did not maintain a precise phase relationship.

\section{EFFECT OF PENDULAR NYSTAGMUS ON VISUAL} ACUITY All patients were distressed by the oscillopsia which they experienced as a result of their pendular nystagmus and this was more or less proportional to the amplitude of the eye movement. We were, however, surprised that visual acuity in patients with pendular nystagmus did not seem to be particularly low when tested using a Snellen chart, as estimated by comparison of acuities measured before and after treatment with hyoscine to suppress the nystagmus, acuity was reduced by only one to two lines of the Snellen chart. The degree of visual impairment 


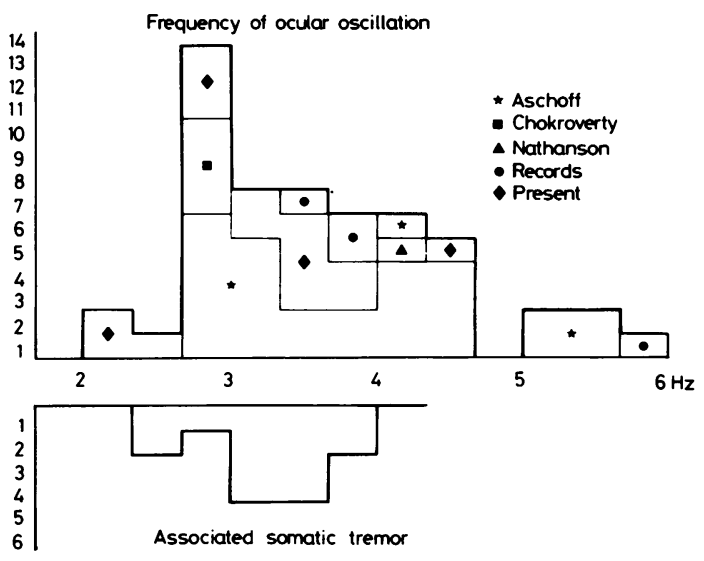

Fig 2 Frequency histograms of the frequencies of pendular nystagmus and associated somatic tremor in our own patients and in patients reported by other authors and those documented in the records of the National Hospital. which our patients experienced seemed more the result of oscillopsia than of loss of acuity.

Severe problems were encountered when the patients attempted to read when it became evident that the nystagmus interfered with the generation of normometric scanning steps. The mechanism appeared to be one of simple summation. When the patient attempted to generate a saccade the amplitude was modified by the addition of the involuntary oscillation which could either reduce or augment the saccade depending upon the phase of the nystagmus.

\section{BEHAVIOURAL CHARACTERISTICS}

As can be seen from the column in the table listing modifying factors in the pendular nystagmus manifest in our patients there were no consistent behavioural characteristics which could be helpful in determining the underlying aetiology. In particular we did not observe any nystagmus to be inhibited following saccadic eye movements as reported by
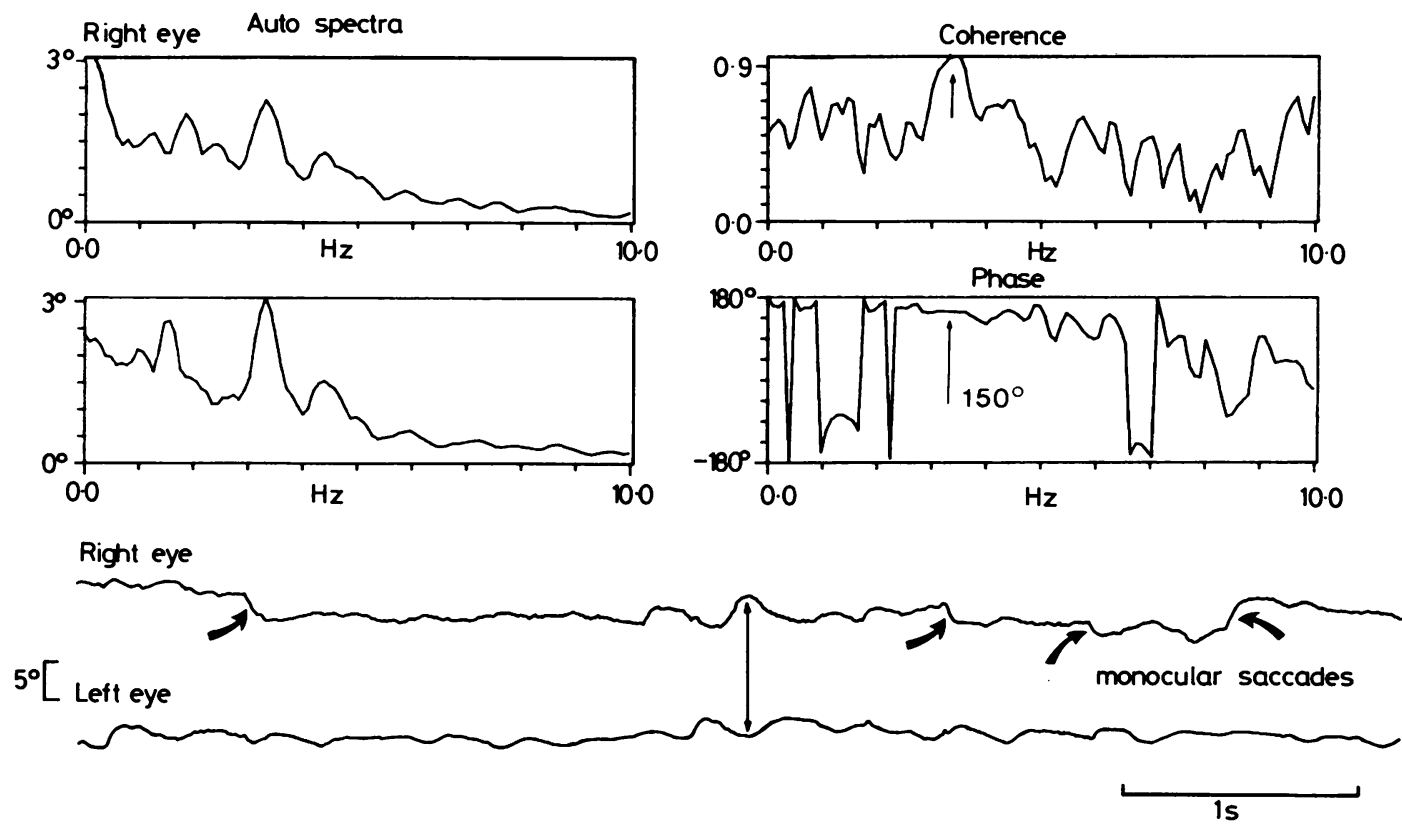

Fig 3 Binocular pendular nystagmus in the horizontal plane recorded from a 28-year-old female patient with a presumed cerebrovascular accident. The movements of her eyes were in the direction of convergence and divergence. The auto spectra indicate the common dominant frequency of the pendular movements. The coherence and phase measurements at this frequency (indicated by small vertical arrows) show that the movements are completely synchronous with a phase difference of $150^{\circ}$. The raw data records show two types of spontaneous involuntary activity. The pendular nystagmus which is common to both eyes as indicated by the synchronisation arrow and monocular saccadic movements of the right eye. The spectral measurements were based on averages derived from ten $10.24 \mathrm{~s}$ samples of data. On this number of averages a coherence of 0.7 was significant at the $2 \%$ level. 


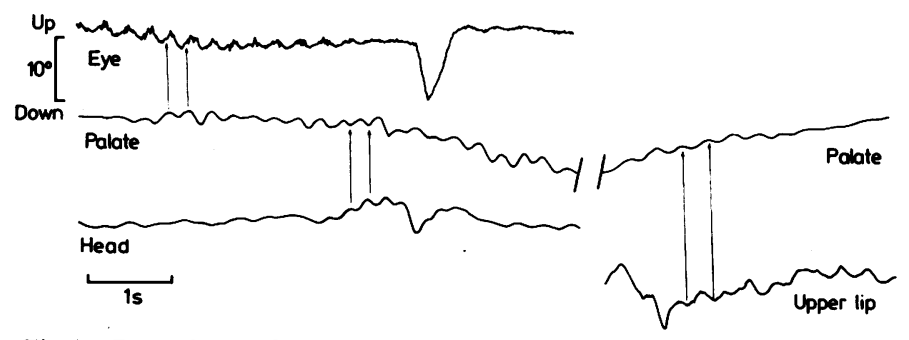

Fig 4 Recordings of somatic tremor and pendular nystagmus taken from a 24-year-old male patient with multiple sclerosis. Synchronisation arrows between the traces indicate that the movements are similar in frequency in all body parts.

Aschoff $e t a l .^{2}$ On the contrary we found a curious instance of an episodic pendular nystagmus in a 24-year-old male patient with multiple sclerosis which was provoked by saccadic mavements of small amplitude as illustrated in fig 5 . With attempts to hold the eyes still there were short bursts of oscillatory eye movement. Whenever the patient made small saccades of about $5^{\circ}$ to $10^{\circ}$ in amplitude two or three cycles of nystagmus were initiated which decayed in the form of a damped oscillation. There was an accompanying tremor of the head with comparable behavioural characteristics. The crescendo-decrescendo appearance and the initiation by sudden movement of such rhythmical involuntary movements in this patient are reminiscent of "startle myoclonus" reported in children."

It has been said that acquired pendular nystagmus in multiple sclerosis is present only during fixation and ceases with eye closure. We found this to be the case in only two of our multiple sclerosis patients.

\section{PHARMACOLOGICAL SUPPRESSION OF PENDULAR NYSTAGMUS}

On the basis of the observation that an intravenous dose of hyoscine can temporarily suppress low frequency somatic tremor in certain cases of multisystem degeneration, ${ }^{9}$ we investigated the effect of this drug in five patients with pendular nystagmus. Four of these had multiple sclerosis and one a brain stem cerebrovascular accident. In all five, 10 minutes after a single intravenous dose of hyoscine $\mathbf{0 . 4} \mathrm{mg}$, the nystagmus was abolished (as judged by observation and electro-oculography). The patients themselves were aware of this and no longer experienced oscillopsia. In one case visual acuity improved from $6 / 12$ to $6 / 6$. The nystagmus recurred after approximately 15 minutes. The patients subsequently noted slight drowsiness but did not experience this during the period when the nystagmus was absent. The potential long term therapeutic application of hyoscine in such cases is being further investigated.
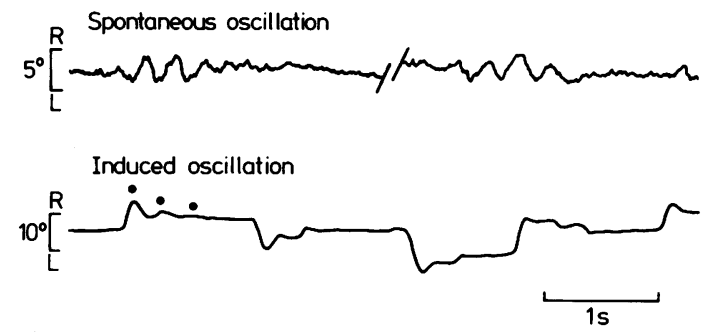

Fig 5 Bursts of pendular nystagmus recorded from a 24-year-old male patient with multiple sclerosis. When the patient tried to keep his eyes still there were bursts of crescendo/decrescendo pendular oscillations as in the upper traces. Execution of small saccades appeared to trigger off two or three cycles of nystagmus as shown in the lower traces.

In several other patients, a range of drugs including leva-dopa, baclofen, clonazepam, prochlorperazine, carbamazepine, tetrabenazine and a variety of major tranquillizers were administered to no avail. Informed consent following a full explanation of the procedure was obtained from all patients with whom pharmacological suppression of nystagmus was attempted.

ASYMMETRICAL FEATURES OF THE WAVE FORM OF PENDULAR NYSTAGMUS

The electro-oculographic technique which was the only eye movement recording method available to us is not sensitive enough to transduce the wave form of pendular nystagmus of small amplitude with a signal to noise ratio adequate to reveal the shape of the movement trajectory in detail. Information about precise trajectory would be useful because it would indicate whether the muscular activity responsible was reciprocally organised or asymmetrical in time or amplitude, which could give clues as to the origin of the oscillation. However, two observations derived from electro-oculographic recordings did suggest that in some cases the trajectory of the movement was asymmetrical indicating that 
either the rhythm of the agonist/antagonist activity was iambic or that active myogenic displacement was occurring in only one direction with movement in the opposite direction being under the influence of the passive elastic properties of orbital tissue.

The first of these observations was that the shape of the wave form of the nystagmus tended to be "saw tooth" rather than sinusoidal; the second was that in some cases the pendular nystagmus exerted a directional bias on other oculomotor responses. This latter point is illustrated in fig 6(a). The recordings are taken from a 25-year-old male patient with a four year history of multiple sclerosis. He had a rotatory nystagmus of the right eye with a torsional pendular movement of the left. The movements of his right eye in response to optokinetic simulation (OKN) using a rotating drum which provided full field stimulation were markedly deranged with drum rotation to the right and relatively preserved with rotation to the left. His responses to caloric and rotational stopping stimuli were similarly affected. The movements of his left eye were normal. Smooth pursuit movements of the right eye were similarly asymmetrical with the oscillations tending to add to the pursuit movement in the rightwards direction giving a hypermetric appearance and to subtract from the pursuit in the leftwards direction giving a hypometric appearance. Corresponding movements

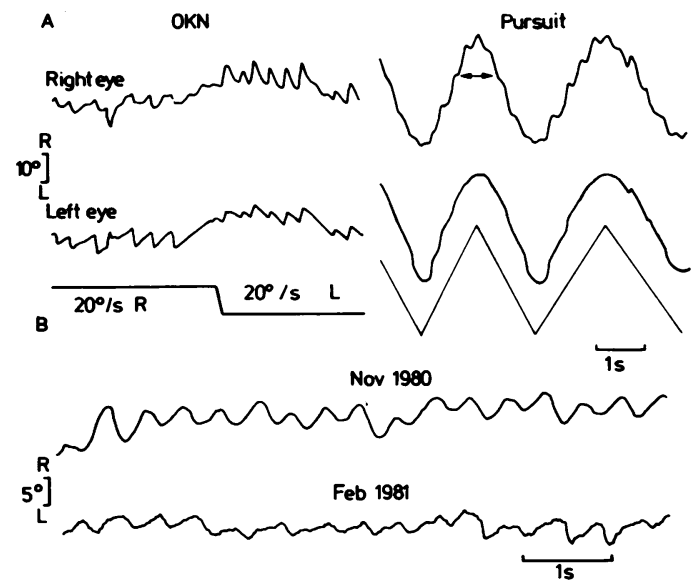

Fig 6 (a) recordings taken from a 25-year-old male patient with multiple sclerosis with a monocular pendular nystagmus of the right eye. The nystagmus interferes with optokinetic nystagmus to the right producing a deranged response. Nystagmus to the left and nystagmus in the good eye is unaffected. Smooth pursuit in the right eye was more affected with rightwards than with leftwards movements (arrow). (b) successive records taken at a 4 month interval from a 49-year-old female patient with a cerebrovascular accident showing an alteration in the nystagmus wave form from pendular to triangular. of the left eye showed a mild pursuit deficit. The shape of the pendular movements of the right eye when the patient was trying to keep his eyes still suggested that there was a stronger pull to the right than to the left. If there were such an asymmetry of muscle action then it would explain the directional characteristics of the patient's pursuit, vestibular and optokinetic responses.

Further evidence for asymmetry in the mechanism of some pendular nystagmus is derived from another of our patients; a 49-year-old woman with a brain stem cerebrovascular accident who presented originally with a pendular nystagmus and other signs of a brain stem lesion. Over the course of four months the signs in her upper limbs partially remitted. Concurrently the pendular nystagmus became a "jerk" nystagmus as illustrated in fig 6 (b).

The implications of these observations upon the interpretation of the results of caloric and rotational tests are important. The possibility of an asymmetrical wave form in pendular nystagmus should be taken into account in interpretation of such tests. Failure to do so could easily lead to a misleading clinical interpretation.

\section{Discussion}

PHYSIOLOGICAL AND ANATOMICAL

CONSIDERATIONS CONCERNING THE NATURE OF PENDULAR NYSTAGMUS

Aschoff $e t a^{2}$ postulated that a lesion in the cerebellar nuclei or their connections was the cause of acquired pendular nystagmus. Their reasons for this included the fact that amongst their patients with pendular nystagmus (all of whom had multiple sclerosis); many had signs of cerebellar nuclear lesions in their upper limbs, for example postural or intention tremor. In addition they reviewed the evidence concerning the site of lesions in oculo-palatal myoclonus which consistently include the dentate and inferior olivary nuclei and brachium conjunctivum. ${ }^{7}{ }^{10}$ They interpreted the pendular eye movements as refiecting loss of postural control of the eyes by the cerebellar nuclei. ${ }^{12}$ There are several reasons why this view is difficult to maintain. Firstly, as emphasised by Castaigne et al, ${ }^{3}$ the existence of monocular pendular nystagmus (in the case of Castaigne's patient a monocular circumduction nystagmus, that is, rotatory nystagmus) implies that the mechanism responsible for generating the oscillation must have access to an oculomotor nucleus or nuclei which innervate only one eye. Although the interpretation of stimulation experiments is difficult because of the possibility of the excitation of axon collaterals, the evidence derived from animal and human studies indicates that electrical stimulation of 
the cerebellum, its nuclei or efferent pathways results in binocular movements. ${ }^{111314}$ Secondly, it is imprudent to argue that the coexistence of pendular nystagmus and somatic tremor in diseases with multifocal lesions implies that these signs arise from the same anatomical lesion. This point is highlighted by the fact that amongst the patients in Aschoff's survey there was by no means a one to one correspondence between cerebellar signs in the upper limbs and the occurrence of pendular nystagmus and that of our own patients less than half had unequivocal signs of cerebellar disease.

The unique feature of pendular nystagmus which requires explanation is that it can be in any plane or combination of planes in either eye, simultaneously and at the same frequency. An important clue as to the nature of its mechanism is that all other saccadic and slow phase oculomotor mechanisms except those responsible for conjugate gaze and vergence may be intact.

Because the generation of saccadic, pursuit, optokinetic and vestibulo-ocular reflex eye movements may be intact in the presence of pendular nystagmus the causal mechanism is unlikely to be related to these major oculomotor systems. The fact that a compound pendular nystagmus may exist in which the movements in various planes are perfectly synchronised (as indicated by a value of unity in the coherence function), implies that the nystagmus arises from disorder in a mechanism which is normally responsible for the co-ordination of eye movement in more than one plane.

In any one eye movement all extra-ocular muscles are involved. ${ }^{15}$ In order to ensure accurate and conjugate gaze, corrections have to be made for deviations from the desired eye position or trajectory which arise from the secondary actions of muscles and changes in the passive visco-elastic properties of orbital tissue. Although not anatomically identified, it is accepted that a neuronal mechanism responsible for such corrections exists. ${ }^{15}$ An important aspect of such a mechanism(s) is that it frequently has a different yet co-ordinated action on the two eyes, particularly as far as the secondary action of the muscles are concerned. This type of co-ordinating mechanism is a candidate for involvement in pendular nystagmus for the consequences of damage to such a structure could explain the variety and synchronicity of eye movements encountered in the disorder.

An anatomical localisation of the lesion responsible for pendular nystagmus in the proximity of the oculomotor nuclei is suggested by several features of the nystagmus and by frequently associated abnormalities of other ocular movements. Stimulation of most supra-nuclear oculomotor centres results in binocular movement and it is only close to or at the level of the oculomotor nuclei that a monocular response is evoked. ${ }^{15}$ It is possible that the abnormal signals responsible for the oscillation have access to motor neurones innervating reciprocally related muscles because they produce a sinusoidal movement in the absence of globe retraction. In order for this to occur the signals must arise at a supranuclear level. However, in the case of a monocular movement this must be near the third nerve nucleus.

These arguments must almost necessarily hold for a monocular nystagmus; however, they have no application in the localisation of a lesion responsible for a conjugate binocular pendular nystagmus. Further evidence that the lesion responsible for pendular nystagmus is near the oculomotor complex is to be inferred from the universal occurrence amongst our patients of associated internuclear ophthalmoplegia or skew deviation, failure of convergence and squint, all of which are signs of derangement between or close to the level of the oculomotor nuclei.

The occurrence of pendular nystagmus in monocular amblyopia with strabismus requires further explanation because there is no evidence of structural damage in the brain stem. However, the character of the nystagmus in these patients is indistinguishable from that seen in multiple sclerosis, for example. The feature common to both is a marked failure in the vergence system and this may indicate that this is an aetiological factor. Certainly the similarity between nystagmus of an amblyopic eye and that resulting from structural lesions of the nervous system strongly suggests that they result from abnormalities of function of the same nervous mechanism. When pendular nystagmus arises in amblyopia we suspect there is additional, compounding insult to the nervous system, for example, migraine.

One can only speculate on the origin of the frequency characteristics of pendular nystagmus. Clues as to the origin of the rhythm come from the fact that associated somatic tremors sometimes have a similar periodicity. It is unlikely that such periodicity is caused by time delays around a form of control loop because the control parameters required for body parts with such diverse functions and physical properties as the eye, tongue or arm must be very different. The uniformity of frequency encountered in the syndrome of oculo-palatal-laryngealdiaphragmatic myoclonus suggests that the rhythmicity derives from properties which are held in common by different types of neurones in different neuronal circuits. As such these properties must be fundamental to the function of the neurone, either in isolation or in the context of its local circuitry. Cycles of excitation and inhibition arising from the 
properties of the neuronal membrane have been described for example in the inferior olive by Llinás and Yarom $^{16}$ and this structure is implicated in the syndrome of oculo-palatal myoclonus. ${ }^{710}$ None of the tremulous movements in the syndrome are necessarily associated with weakness or ataxia of the affected part which implies that the primary motor pathway is intact so that the tremor must arise from an accessory pathway.

\section{CLINICAL ASPECTS OF PENDULAR NYSTAGMUS}

In general the prognosis for cessation of involuntary ocular movement and diminution of oscillopsia is poor. The results of pharmacological intervention so far have been discouraging. In those cases where the nystagmus has been modified by drug treatment (for example, with barbiturate and hyoscine) the subsequent level of impairment of concentration would be unacceptable for everyday life.

\section{References}

${ }^{1}$ Guillain G. The Syndrome of Synchronous and Rhythmic Palato-Pharyngo-Laryngo-Oculo-Diaphragmatic Myoclonus. Proc $R$ Soc Med 1938;31:1031-8.

${ }^{2}$ Aschoff JC, Conrad B, Kornhuber HH. Acquired Pendular Nystagmus with oscillopsia in Multiple Sclerosis: a sign of cerebellar nuclei disease. J Neurol Neurosurg Psychiatry 1974;37:570-7.

${ }^{3}$ Castaigne P, Chain F, Pierrot-Deseiligny C, Larmande $P$. Le nystagmus de circumduction monoculair. Etude clinique, oculographique et électro-myographique d'un cas, dans la sclérose en plaques Rev Neurol (Paris) 1979;135:51-7.

4 Findley LJ, Gresty MA, Halmagyi GM. A novel method of recording arm movements: A survey of common abnormalities. Arch Neurol 1980;38:38-42.

5 Hewlett Packard. Digital Signal Analyser User's Guide. HP 05420-900 32, July, 1978.

- Chokroverty S, Barron KD. Palatal myoclonus and rhythmic ocular movements: A polygraphic study. Neurology (Minneap) 1969;19:975-82.

${ }^{7}$ Nathanson M. Palatal myoclonus. Further clinical and Pathophysiological observations. Arch Neurol Psychiatry 1956;75:285-96.

' Gresty MA, Halmagyi GM. Abnormal Head Movements. J Neurol Neurosurg Psychiatry 1979;42:70514.

9 Ordenstein L. Sur la paralysie agitante et la sclérose en plaques généralisée. Paris: Delahayet, 1868, 32 (ref to M Charcot).

${ }^{10}$ Bender MB, Nathanson M, Gordon GG. Myoclonus of muscles of the eye, face and throat. Arch Neurol Psychiatry 1952;67:44-58.

"Nashold BS, Slaughter DG, Gills JP. Ocular reactions in man from deep cerebellar stimulation and lesions. Arch Ophthalmol 1969;81:538-43.

${ }^{12}$ Kornhuber HH. Motor functions of cerebellum and basal ganglia: the cerebellocortical saccadic (ballistic) clock, the cerebellonuclear hold regulator, and the basal ganglia ramp (voluntary speed smooth movement) generator. Kybernetik 1971;8:157-62.

${ }^{13}$ Nashold BS, Gills JP. Ocular signs from brain stimulation and lesions. Arch Ophthalmol. 1967;77:609-18.

14 Ron S, Robinson DA. Eye movements evoked by cerebellar stimulation in the alert monkey. $J$ Neurophysiol 1973;36:1004-22.

${ }^{15}$ Bender MB. Brain control of conjugate horizontal and vertical eye movements. A survey of the structural and functional correlates. Brain 1980;103:23-69.

${ }^{16}$ Llinás RR, Yarom Y. Electrophysiological properties of mammalian inferior olivary cells in vitro. In: Courville J, de Montigny C, Lemarre Y, eds. The Olivary Nucleus. Anatomy and Physiology. New York: Raven Press, 1980:379-88. 